\title{
ANÁLISE DA PERMEAÇÃO DE NANOLIPÍDEOS EM CABELO HUMANO
}

\author{
Ivana M. B. Gomes ${ }^{1}$ \\ Aline Priscila Campos Pereira ${ }^{2}$ \\ Michely Glenda Pereira da Silva ${ }^{3}$ \\ Taciana D. Magrini Alva ${ }^{4}$ \\ Airton A. Martin ${ }^{5}$
}

Resumo: Com a descoberta das nanopartículas as indústrias de cosméticos capilares buscaram investir em produtos com esta nova tecnologia, já que há uma grande demanda por novos produtos e resultados mais efetivos nesta área. Para ser eficiente, é necessário que um produto hidratante de cabelo permeie as camadas da fibra capilar, requisito esperado nas nanopartículas de lipídeos, ou nanolipídeos. A espectroscopia confocal Raman é uma técnica de espectroscopia vibracional sensível para as análises de amostras biológicas devido à especificidade química inerente de frequências de vibração do espectro, porém a sua capacidade de analisar as fibras do cabelo permanece pouco estudada. O objetivo deste trabalho foi utilizar a técnica não invasiva de Espectroscopia Raman Confocal para adquirir espectros correspondentes à permeação de nanolipídeos no fio de cabelo humano. Neste a capacidade da técnica para gerar informações sobre a localização de um nanolipídeo nas fibras de cabelo.

Palavras-chave: Cabelo; Permeação; Espectroscopia Raman Confocal; Nanolipídeo.

\footnotetext{
1 Graduanda em Engenharia química pela Universidade do Vale do Paraíba, Brasil. Iniciação Científica no Laboratório de Espectroscopia Raman Confocal/Instituto de Pesquisa e Desenvolvimento, Brasil. E-mail: ivanabuck@hotmail.com.

2 Graduação em Ciências Biológicas pela Universidade do Vale do Paraíba, Brasil. Iniciação Científica no Laboratório de Espectroscopia Raman Confocal/Instituto de Pesquisa e Desenvolvimento, Brasil. E-mail: allinneferrara@gmail.com.

${ }^{3}$ Enfermeira, Mestre em Engenharia Biomédica pela Universidade do Vale do Paraíba - UNIVAP. Doutoranda em Engenharia Biomédica - Bolsista da Coordenação de Aperfeiçoamento de Pessoal de Nível Superior CAPESInstituto de Pesquisa e Desenvolvimento - IP\&D/Universidade do Vale do Paraíba - UNIVAP, Brasil. E-mail: michely.glenda@hotmail.com.

4 Doutorado em Nanociências e Materiais Avançados pela Universidade Federal do ABC, Brasil(2015) Pós Doutorado da Universidade do Vale do Paraíba, Brasil. E-mail: texying@gmail.com.

5 Bolsista de Produtividade em Pesquisa 1D - CAEE, Pós-doutorado pelo Instituto Max Planck for Festokoperforschung - Stuttgart (1996-1999). Professor adjunto da Universidade do Vale do Paraíba. Graduado em Física pela Universidade Estadual de Londrina (1985), Mestre em Física pela Universidade de São Paulo (1988), Mestre em Ciências pela University of Toronto - Canada (1991). de ensino, pesquisa e extensão. Coordenador do Laboratório de Espectroscopia Vibracional Biomédica e Lab. PROBES- Desenvolvimentos de Projetos em Biomedicina e Engenharias, Brasil. E-mail: amartin@univap.com.br.
} 